\title{
EU QUESTIONNAIRE TO SCREEN FOR OBSTRUCTIVE SLEEP APNOEA VALIDATED IN SLOVAKIA
}

\author{
Eva Feketeová1, Imrich Mucska², Katarina Klobučníková ${ }^{3}$, Soňa Grešová4, Judita Stimmelová4, Ivana Paraničová5, \\ Ivana Trojová ${ }^{5}$, Jaroslav Rosenberger ${ }^{6}$, Kvetoslava Rimárová ${ }^{7}$, Erik Dorko ${ }^{7}$ \\ ${ }^{1}$ Department of Neurology, Faculty of Medicine, Pavol Jozef Šafárik University in Košice and Louis Pasteur University Hospital, Košice, Slovak \\ Republic \\ ${ }^{2}$ Outpatient Clinic for Sleep-Disordered Breathing, University Hospital Bratislava, Bratislava, Slovak Republic \\ ${ }^{3} 1$ st Department of Neurology, Faculty of Medicine, Comenius University in Bratislava, Bratislava, Slovak Republic \\ ${ }^{4}$ Department of Medical Physiology, Faculty of Medicine, Pavol Jozef Šafárik University in Košice, Košice, Slovak Republic \\ ${ }^{5}$ Department of Pneumology and Phtiseology, Faculty of Medicine, Pavol Jozef Šafárik University in Košice and Louis Pasteur University Hospi- \\ tal, Košice, Slovak Republic \\ ${ }^{6}$ Department of Health Psychology, Faculty of Medicine, Pavol Jozef Šafárik University in Košice, Košice, Slovak Republic \\ ${ }^{7}$ Department of Public Health and Hygiene, Faculty of Medicine, Pavol Jozef Šafárik University in Košice, Košice, Slovak Republic
}

\section{SUMMARY}

Objective: Obstructive sleep apnoea syndrome (OSAS) associated with daytime sleepiness (DS) contributes to a higher incidence of motor vehicle accidents. Validation of fitness to drive in driving license applicants, with special concern regarding OSAS accompanied by excessive DS, became mandatory under new EU legislation in January 2016. The aim of the study was to translate and validate the recommended questionnaire to screen for OSAS (Q-OSAS) in the Slovak population. No data on any Q-OSAS validation has previously been published.

Methods: The translated Q-OSAS was administered to 311 Slovak patients prior to a planned overnight polysomnography. The diagnostic accuracy of the Q-OSAS in OSAS with an apnoea-hypopnoea index of 15 or more/h of sleep was evaluated by calculating the area under the ROC curve.

Results: The sensitivity and specificity of the cut-off at 10 points for the Q-OSAS was $57 \%$ and $67 \%$, respectively, with an increase of sensitivity and a decrease of specificity with a lowering of the cut-off values. Excluding the Epworth Sleepiness Scale (ESS) score from the final statistics yielded the best sensitivity $(77 \%)$, specificity $(50 \%)$, and an area under the ROC curve $(0.637)$ for the cut-off value of 8 points (an equivalent of 10 points with the full version of the Q-OSAS).

Conclusion: The Q-OSAS is an appropriate screening tool to facilitate the screening of subjects potentially at risk from moderate and severe OSAS. A modified two-step interpretation of the Q-OSAS in Slovakia yielded the best sensitivity, and in the future could promote evaluation of sleepiness in sleep and wake disorders other than OSAS for fitness to drive.

Key words: OSAS, screening questionnaire, ESS, daytime sleepiness, fitness to drive

Address for correspondence: E. Feketeová, Department of Neurology, Faculty of Medicine, Pavol Jozef Šafárik University in Košice and Louis Pasteur University Hospital, Trieda SNP 1, 04011 Košice, Slovak Republic. E-mail: eva.feketeova@upjs.sk

https://doi.org/10.21101/cejph.a5278

\section{INTRODUCTION}

Obstructive Sleep Apnoea (OSA) syndrome is a prevalent chronic sleep disorder frequently associated with cardiovascular comorbidity and associated with several adverse clinical outcomes. Persons with OSA are at a high risk of being involved in occupational accidents (1), and have an increased risk of motor vehicle accidents (MVAs) because of their impaired vigilance (2). The documented increased risk of MVAs in OSAS patients resulted in a new legislative process and requirements for validation of fitness to drive in driving license applicants with specific concern regarding OSAS (3).

New EU legislation has been passed on fitness-to-drive tasks for the selection and treatment of drivers with an apnoea-hypo- pnoea index (AHI) of 15 or more per hour of sleep in the presence of excessive daytime sleepiness (DS). Night polysomnography, considered the "gold standard" for OSA diagnosis, is not widely available and its expense constitutes a burden on public health resources. Therefore, several questionnaires calculating anthropometric data, sex and age and asking more or fewer questions about the presence of snoring, lapses of breathing during the night, daytime spells, arterial hypertension and other symptoms have been developed and evaluated for the presence of OSA with an AHI of 15 or more with various specificities and sensitivities: the Berlin questionnaire (4-7), the STOP Questionnaire (8), the STOP-Bang Questionnaire $(7,9)$, the ASA Checklist (7), the Wisconsin questionnaire $(5,10)$ and the Sleep Apnoea of Sleep Disorders Questionnaire (11). 
The Obstructive Sleep Apnoea Working Group under European Commission Directive 2006/126 proposed a Questionnaire to screen for OSAS (Q-OSAS) in order to identify driving license applicants at risk from OSAS. The questionnaire has not been made mandatory, but member states are strongly encouraged to make use of it during screening (3).

The aim of the study was to translate and validate Q-OSAS in the Slovak population and to test it for OSAS diagnostic prediction, all the more so because it has not been validated in any other population.

\section{MATERIALS AND METHODS}

\section{Patients and the Study Protocol}

A total of 311 subjects was recruited from sleep labs in Slovakia between November 2014 and December 2015 during the validation process of the Slovak version of Q-OSAS. All subjects agreed to participate and provided written informed consent prior to the study, which was approved by Local Ethics Committees.

The subjects, who were all aged 18 years or older were scheduled for full-night polysomnography (PSG) on the basis of having experienced a snoring spell, disrupted sleep or being referred because of arterial hypertension. They were asked to fill in the Slovak version of Q-OSAS just before the PSG examination. The final Q-OSAS score was then calculated. Full-night PSGs were performed in accredited sleep labs and scored by certified sleep physicians. The PSG consisted of a frontal and/or central and occipital electroencephalogram, a right and left electrooculogram and a submental electromyogram; airflow and respiratory effort were determined by measurement of chest and abdominal motion, arterial oxygen saturation, ECG and $\mathrm{m}$. tibialis anterior electromyogram. Sleep was manually scored according to the American Academy of Sleep Medicine (12). Respiratory events were scored as apnoeas (absence of airflow $\geq 10 \mathrm{~s}$ : obstructive apnoeas in the presence of respiratory effort; central apnoeas in the absence of respiratory effort), and hypopnoeas (decrease of flow to $>50 \%$ below baseline $\geq 10 \mathrm{~s}$ associated with decreased $\mathrm{SaO} 2>3 \%$ or with arousal). The AHI was computed as the total number of apnoeas and hypopnoeas divided by the total sleep time in hours.

The questionnaires and the AHI were collected the next morning. Partially missing data $(n=23)$ were excluded from the analysis. A total of 288 subjects remained for analysis.

\section{Q-OSAS}

The Q-OSAS consists of eleven items. The first five are objective items - gender, age, weight and height, and the existence of recent MVAs involving injuries or property damage that required police reports. The 6-10th items deal with the frequency and intensity of snoring, the presence of breathing pauses as identified by bed partners or witnesses, the restorative quality of sleep and the presence of arterial hypertension.

The last item of the questionnaire searches for the presence of DS using the Epworth Sleepiness Scale (ESS) (13). The ESS is an 8-item questionnaire with 4 possible answers per question expressing the probability of falling asleep, rated from 0 to 3 .
A total of 24 points is the maximum sleepiness level. Subjects without DS have an ESS score up to 10; a value of 11 is considered to be mildly abnormal DS, whereas a score of 15 or higher is considered as severe somnolence.

The interpretation of Q-OSAS was based on an attributed value for each question, reflecting the risk of MVAs or the possibility of suffering from OSAS, as well as the level of uncertainty. This value was based on a consensus minimal agreement among the members of the Obstructive Sleep Apnoea Working Group (Appendix 1). A score of 10 points or more was considered as a positive result (3).

\section{Translation of the Q-OSAS}

The Q-OSAS was forward and backward translated by two independent teams of investigators involved in further study data collection and two independent translators with no awareness of the measured concepts, all fluent in English and Slovak. The results of the translations were later discussed for semantic and idiomatic issues and tested on a sample of 20 patients, which led to the raising of two minor issues included in the final translation.

\section{Statistics}

Descriptive statistics were used to describe demographic features: age, sex, and BMI, Q-OSAS score, AHI and ESS scores. Differences between AHI groups were tested by $\chi^{2}$-test or Mann-Whitney U-test, as appropriate.

Receiver operating characteristic (ROC) curves were used:

a) to assess how a Q-OSAS score of 10 points or more can predict an AHI score of 15 and more,

b) to compare the sensitivity and specificity of Q-OSAS for different cut-off values of the Q-OSAS score,

c) to compare the sensitivity and specificity of Q-OSAS without the ESS score included in the final score.

\section{RESULTS}

A total of 311 subjects referred for polysomnography completed the Q-OSAS. Complete data were obtained from 288 subjects $(92.6 \%)$ and used for analysis. Subject characteristics (age, sex, BMI, Q-OSAS score, and AHI) are listed in Table 1. Age, sex, BMI and ESS scores significantly varied in the group of patients with an AHI of less than 15 and the group with 15 or more. A total of 216 patients had an AHI of 15 or more with a median score on the Q-OSAS of 10. Patients with an AHI below 15 had a median Q-OSAS score of 8 .

The specificity and sensitivity of the Q-OSAS (Table 2) was calculated for different cut-off values for the score:

A) recommended 10 points or more as a positive result (3),

B) used 9, 8, 7,6 cut-off values for the Q-OSAS score,

C) used cut-off values for the questionnaire without an ESS score: 8, 7, 6, 5, 4 (adopted for exclusion of ESS).

The sensitivity and specificity of the cut-off at 10 points (A) was $57 \%$ and $67 \%$, respectively, with an increase of sensitivity and decrease of specificity with a lowering of the cut-off values (B). Excluding the ESS score from the final statistics yielded the best sensitivity (77\%) and specificity (50\%) for the cut-off value 
Table 1. Subjects characteristics $(N=288)$

\begin{tabular}{|c|c|c|c|c|}
\hline & Total & $\mathrm{AHI}<15$ & $\mathrm{AHI} \geq 15$ & \multirow{2}{*}{$\mathrm{p}$-value } \\
\hline & $N=288$ & $n=72$ & $\mathrm{n}=\mathbf{2 1 6}$ & \\
\hline Age (year) & $53(17)$ & $48.5(19)$ & $54(15)$ & 0.009 \\
\hline Sex (female/male) & $63 / 225(21 / 79 \%)$ & $26 / 46(36 / 64 \%)$ & $37 / 179(17 / 63 \%)$ & $<0.001$ \\
\hline BMI $\left(\mathrm{kg} / \mathrm{m}^{2}\right)$ & $31(7)$ & $28.5(6)$ & $32(7)$ & $<0.001$ \\
\hline Q-OSAS score & $10(4)$ & $8(5)$ & $10(4)$ & $<0.001$ \\
\hline ESS score & $8(7)$ & $8(8)$ & $10(6)$ & 0.003 \\
\hline AHI (per hour of sleep) & $31.7(44.7)$ & $6.1(7.3)$ & $54(39.7)$ & \\
\hline
\end{tabular}

Data show median and (IQR) values; BMI - body mass index, Q-OSAS score - Questionnaire to screen for OSAS score, AHI - Apnoea-Hypopnoea Index, ESS score Epworth Sleepiness Scale score.

Table 2. Sensitivity, Specificity and ROC - area under the curve for different cut-off values of Q-OSAS score and Q-OSAS score without ESS score

\begin{tabular}{|c|c|c|c|c|c|c|c|}
\hline $\begin{array}{l}\text { Q-OSAS cut-off } \\
\text { score }\end{array}$ & Sensitivity & Specificity & $\begin{array}{l}\text { Area under } \\
\text { the curve }\end{array}$ & $\begin{array}{l}\text { Q-OSAS cut-off } \\
\text { score without ESS }\end{array}$ & Sensitivity & Specificity & $\begin{array}{c}\text { Area under } \\
\text { the curve }\end{array}$ \\
\hline 6 & 0.944 & 0.889 & 0.528 & 6 & 0.926 & 0.861 & 0.532 \\
\hline 7 & 0.880 & 0.750 & 0.565 & 7 & 0.852 & 0.736 & 0.558 \\
\hline 8 & 0.824 & 0.569 & 0.627 & 8 & 0.773 & 0.500 & 0.637 \\
\hline 9 & 0.685 & 0.458 & 0.613 & 9 & 0.593 & 0.361 & 0.616 \\
\hline 10 & 0.565 & 0.333 & 0.616 & 10 & 0.463 & 0.236 & 0.613 \\
\hline
\end{tabular}

Q-OSAS score - Questionnaire to screen for OSAS score, ESS score - Epworth Sleepiness Scale score

of 8 points, the equivalent of a score of 10 points or more with the full version of Q-OSAS (Table 2).

\section{DISCUSSION}

The aim of this study was to validate the translated Q-OSAS questionnaire for the Slovak population and to assess its ability to predict an AHI score. The results of the study showed that the recommended Q-OSAS cut-off among subjects indicated for PSG had a mean sensitivity of $57 \%$ and a specificity of $66 \%$ for selecting a population of drivers at risk from OSAS with an AHI $\geq 15$ in the Slovak population.

A lot of data has been published concerning different questionnaires for screening moderate and severe OSAS (AHI $\geq 15$ ) showing variable predictive parameters and similarities to our data (14).

The STOP-BANG questionnaire was originally validated for OSA screening of surgical patients in preoperative settings (8). The comparison of the STOP-BANG scores to AHI in the similar setting of Sleep Laboratory-referred patients suggests the potential value of the STOP-BANG questionnaire in identifying some individuals who are not likely to have moderate or severe OSA (negative predictive values were 87 , and $96 \%$, respectively). The sensitivity of the STOP-BANG for AHI > 15 and $>30$ was $93 \%$ and $97 \%$, respectively, but with very low specificities of $40 \%$ and $33 \%$ (15).

The Berlin questionnaire was validated in sleep-clinic patients taken from the general population and surgical patients. Its predictive performance for OSA varied greatly among different patient populations. In primary care patients, the sensitivity and specificity were found to be $54 \%$ and $97 \%$, respectively, at a cut-off of AHI greater than 15 (4). In sleep clinic patients, the Berlin questionnaire showed sensitivity of $57 \%$ and specificity of $43 \%$ at a respiratory disturbance index greater than $15(6)$. The predictive value of the Berlin questionnaire was less effective than the STOP-BANG in patients referred for coronary artery bypass grafting versus abdominal surgery (21). The role of comorbid diagnoses in different studied populations was suggested.

Comparative validation of the Berlin questionnaire, the ASA checklist and the STOP questionnaire in the same surgical patients $(7,8)$ found no significant differences between the three screening tools in the predictive parameters. The sensitivity/specificity for AHI $>15$ was $74 \% / 53 \%$ in the STOP questionnaire, $78 \% / 50 \%$ in the Berlin questionnaire and $79 \% / 37 \%$ in the ASA Checklist, respectively, and for AHI $>30$ the sensitivity/specificity were STOP questionnaire: $80 \% / 49 \%$, Berlin questionnaire: $87 \% / 46 \%$ and ASA Checklist: $87 \% / 36 \%$. Compared with the aforementioned studies, our results demonstrated the sensitivity and specificity of Q-OSAS as comparable to the published data.

In the second step of the statistical analysis, we considered a decrease in the cut-off values of the Q-OSAS, increasing the sensitivity at the cost of an unacceptable decrease in the specificity for a screening utility.

The last step of the analysis was testing the validity of Q-OSAS without DS evaluation. We considered the ESS (Question No 11) used for DS evaluation to be the weakest point of the questionnaire, because of the number of variables that can influence ESS validity and the value to predict the OSA alone. In drivers the role of dissimulation of DS caused by fear of losing one's driving license when being asked about the possibility of falling asleep while driving is expected. The ESS shows high sensitivity and specificity 
in the evaluation of sleepiness among patients with central disorders of hypersomnolence correlated with results of the Multiple Sleep Latency Test; however, it shows only moderate correlation with the objective measures in patients with OSAS $(14,15)$. The exclusion of the ESS score from the final score of the Q-OSAS with a cut-off point of 8 in the present study brought very good specificity (77\%) with acceptable sensitivity $(50 \%)$. The results of modified interpretation of the Q-OSAS (ESS excluded from the final statistics) showed results comparable to the aforementioned screening questionnaires. Despite the limited value of ESS in the evaluation of DS in OSAS patients, the ESS is considered to be the gold standard in the evaluation of DS. Furthermore, the presence of excessive DS is a crucial feature for fitness to drive despite its subjective nature in the questionnaire and subjects with an ESS score above 10 should be processed for the further examination in a sleep centre whatever the Q-OSAS score is.

A limitation of the study was that the subjects did not come from the general population of drivers applying for driving licenses but were individuals with disrupted sleep and/or snoring planned for overnight PSG examination (they were not prescreened by oximetry or polygraphy).

\section{CONCLUSIONS}

The study approved Q-OSAS as being the screening tool to facilitate the screening of subjects potentially at risk of moderate and severe OSAS for evaluation of the fitness to drive, and has been validated for the first time. Evaluation of ESS apart of the other questions could increase the potential of Q-OSAS for traffic safety to search for DS accompanying OSAS and also other sleep and wake disorders.

Therefore, in the Slovak population we suggest the two step interpretation of the questionnaire: Subjects with a score of 8 and above (modified interpretation of Q-OSAS) and/or ESS score over 10 have to be recommended for PSG evaluation in the sleep centre.

\section{Funding}

The Operational Program for Research and Development of the Agency of the Ministry of Education of the Slovak Republic for the Structural Funds of the European Union provided financial support in the form of Project CEVA, Contract No. 034/2009/2.1/OPR\&D funding (50\%) and VEGA $1 / 0198 / 13$ a $1 / 0011 / 14$. The sponsors had no role in the design or conduct of this research.

\section{Conflict of Interests}

None declared

\section{REFERENCES}

1 Lindberg E, Carter N, Gislason T, Janson C. Role of snoring and daytime sleepiness in occupational accidents. Am J Respir Crit Care Med. 2001;164(11):2031-5.

2. Young T, Peppard PE, Gottlieb DJ. Epidemiology of obstructive sleep apnea: a population health perspective. Am J Respir Crit Care Med. 2002;165(9):1217-39.

3. McNicholas W, editor. New standards and guidelines for drivers with obstructive sleep apnoea syndrome. Report of the Obstructive Sleep Apnoea Working Group [Internet]. Brussels: European Commission; 2013 [cited 2014 Nov 24]. Available from: https://ec.europa.eu/transport/ road safety/sites/roadsafety/files/pdf/behavior/sleep apnoea.pdf.

4. Netzer NC, Stoohs RA, Netzer CM, Clark K, Strohl KP. Using the Berlin questionnaire to identify patients at risk for the sleep apnea syndrome. Ann Intern Med. 1999;131(7):485-91.

5. Sharma SK, Vasudev C, Sinha S, Banga A, Pandey RM, Handa KK. Validation of the modified Berlin questionnaire to identify patients at risk for the obstructive sleep apnoea syndrome. Indian J Med Res. 2006;124(3):281-90.

6. Ahmadi N, Chung SA, Gibbs A, Shapiro CM. The Berlin questionnaire for sleep apnea in a sleep clinic population: relationship to polysomnographic measurement of respiratory disturbance. Sleep Breath. 2008;12(1):39-45.

7. Chung F, Yegneswaran B, Liao P, Chung SA, Vairavanathan S, Islam $\mathrm{S}$, et al. Validation of the Berlin questionnaire and American Society of Anesthesiologists checklist as screening tools for obstructive sleep apnea in surgical patients. Anesthesiology. 2008;108(5):822-30.

8. Chung F, Yegneswaran B, Liao P, Chung SA, Vairavanathan S, Islam S, et al. STOP questionnaire: a tool to screen patients for obstructive sleep apnea. Anesthesiology. 2008;108 (5):812-21.

9. Boynton G, Vahabzadeh A, Hammoud S, Ruzicka DL, Chervin RD. Validation of the STOP-BANG questionnaire among patients referred for suspected obstructive sleep apnea. J Sleep Disord Treat Care. 2013;2(4). doi: 10.4172/2325-9639.1000121.

10. Young T, Palta M, Dempsey J, Skatrud J, Weber S, Badr S. The occurrence of sleep-disordered breathing among middle-aged adults. N Engl J Med. 1993;328(17):1230-5.

11. Weatherwax KJ, Lin X, Marzec ML, Malow BA. Obstructive sleep apnea in epilepsy patients: the sleep apnea scale of the sleep disorders questionnaire (SA-SDQ) is a useful screening instrument for obstructive sleep apnea in a disease-specific population. Sleep Med. 2003;4(6):517-21.

12. Iber C, Ancoli-Israel S, Chesson A, Quan SF. The AASM manual for the scoring of sleep and associated events: rules, terminology and technical specifications. Westchester: American Academy of Sleep Medicine; 2007.

13. Johns MW. A new method for measuring daytime slepiness: the Epworth Sleepiness Scale. Sleep. 1991;14(6):540-5.

14. Abrishami A, Khajehdehi A, Chung,F. A systematic review of screening questionnaires for obstructive sleep apnea. Can J Anesth/J Can Anesth. 2010;57(5):423-38.

15. Nunes FS, Danzi-Soares NJ, Genta PR, Drager LF, Cesar LA, LorenziFilho G. Critical evaluation of screening questionnaires for obstructive sleep apnea in patients undergoing coronary artery bypass grafting and abdominal surgery. Sleep Breath. 2015;19(1):115-22.

16. Olson LG, Cole MF, Ambrogetti A. Correlations among Epworth Sleepiness Scale scores, multiple sleep latency tests and psychological symptoms. Sleep Res. 1998;7(4):248-53.

17. Benbadis SR, Mascha E, Perry MC, Wolgamuth BR, Smolley LA, Dinner DS. Association between the Epworth Sleepiness Scale and the multiple sleep latency test in a clinical population. Ann Intern Med. 1999;130(4 Pt 1):289-92.

18. Colquhoun CP, Casolin A. Impact of rail medical standard on obstructive sleep apnoea prevalence. Occup Med. 2016;66(1):62-8.

Received December 6, 2017 Accepted in revised form September 19, 2018 
Appendix 1: Proposed Questionnaire to screen for OSAS and its interpretation according to the Obstructive Sleep Apnoea Working Group (3).

\section{Questionnaire for OSAS Screening}
1. Sex
2. Age
3. Weight
4. Height
5. Have you ever dozed off while driving?
YES NO
DON'T KNOW
6. Have you had a serious accident (with personal injuries or property damage)
due to sleepiness in the last 3 years?
YES NO DON'T KNOW
7. Do you usually snore loudly almost every night?
YES NO
DON'T KNOW
8. Have you been told your breathing stops during your sleep?
YES NO
DON'T KNOW
9. Do you usually wake up refreshed after a full night's sleep?
YES NO
DON'T KNOW
10. Do you suffer from, or are being treated for, Arterial Hypertension?
YES NO
DON'T KNOW
11. Please complete the questionnaire on usual daytime sleepiness, called the Epworth Sleepiness Scale, on the next page

\section{Epworth Sleepiness Scale}

How likely are you to doze off or fall asleep in the following situations, in contrast to feeling just tired? Even if you have not done some of these things recently try to work out how they would have affected you. Use the following scale to choose the most appropriate number for each situation:

$0=$ no chance of dozing

$1=$ slight chance of dozing

$2=$ moderate chance of dozing

$3=$ high chance of dozing

\section{Situation}

Sitting and reading

Watching TV

Sitting inactive in a public place (e.g a theatre or a meeting)

As a passenger in a car for an hour without a break

Lying down to rest in the afternoon when circumstances permit

Sitting and talking to someone

Sitting quietly after a lunch without alcohol

In a car, while stopped for a few minutes in traffic

\section{Chance of Dozing}

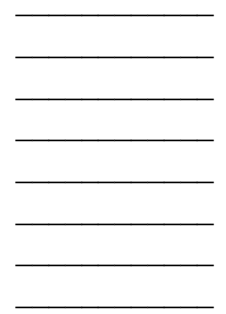

ESS Score:

\section{Interpretation}

Q. 1: Female $=1$; Male $=2$

Q. 2: Age below 30 years $=1$; Age 31 years or above $=2$

Q. 3-4: A BMI below $30 \mathrm{~kg} / \mathrm{m}^{2}=1 ; 31-35 \mathrm{~kg} / \mathrm{m}^{2}=2 ; 36 \mathrm{~kg} / \mathrm{m}^{2}$ or higher $=3$

Q. 5: A positive answer $=3$; negative answer $=0$; don't know $=2$

Q. 6: A positive answer $=4$; negative answer $=0$; don't know $=3$

Q. 7: A positive answer $=2$; negative answer $=0$; don't know $=1$

Q. 8: A positive answer $=1$; negative answer $=0$; don't know $=0$

Q. 9: A negative answer $=2$; positive answer $=0$; don't know $=1$

Q. 10: A positive answer $=2$; negative answer $=0$; don't know $=1$

ESS: From 11 to $14=2 ; 15$ or higher: 4

If the result is 10 or higher, screening is defined as positive. 